\title{
O USO DO VDC SCORECARD NA VALIDAÇÃO DE MÉTODOS PARA ANÁLISE DE DESEMPENHO DA GESTÃO DO PROCESSO DE PROJETO NO CENÁRIO BRASILEIRO
}

Use of VDC scorecard in the validation of methods for analysis of performance in the design process management in the brazilian scene

\author{
Saulo Britto', Sérgio Scheer ${ }^{1}$, Calvin Kam², Martin Fischer ${ }^{2}$
}

RESUMO A implementação de tecnologias da informação na construção civil, como Virtual Design and Construction/Building Information Modeling (VDC/BIM), representam uma nova perspectiva para melhores práticas na Arquitetura, Engenharia, Construção, Operação (AECO). Contudo, no cenário brasileiro, essas práticas carecem de métodos para medição e análise de desempenho processual da informação na gestão do processo de projeto, demonstrando a deficiência da documentação de critérios e seleção de indicadores que contribuam para melhoria do setor da construção civil. Trata-se de uma pesquisa descritiva sobre a exploração do método VDC Scorecard e a adaptação à realidade brasileira, tendo por base pesquisa bibliográfica e documental existentes em bases de dados internacionais e nacionais, explorando os seguintes aspectos: benefícios registrados; dificuldades na implementação; boas práticas na gestão do projeto. A pesquisa valida a importância dos métodos de desempenho que se adequem ao contexto nacional, viabilizando a integração e controle da informação entre agentes envolvidos num modelo colaborativo de edificação virtual e apropriação de dados por meio dos modelos de desempenho configurados pela análise contínua dos processos, da organização e do produto. Essa compreensão possibilita a documentação de um banco de dados de práticas eficientes para gestão de processos de projeto configurando o desempenho do modelo.

PALAVRAS-CHAVE: Análise de desempenho VDC/BIM, VDC Scorecard, Gestão do processo de projetos

ABSTRACT The implementation of information technologies for civil construction such as Virtual Design and Construction/Building Information Modeling (VDC/BIM) represents a new perspective on better practices in the Architecture, Engineering, Construction, and, Operation (AECO). However, in the Brazilian scene, these practices are need methods for measuring and process performance analysis of the information in the design process management, demonstrating the lack of criteria documentation and indicators selection that contribute to the improvement in the civil construction sector. It is a descriptive research exploring the VDC Scorecard method and its application to Brazilian reality, based on existing literature and documentary research in international and national databases, exploring the following aspects: registered benefits; difficulties for implementation; and good practices in project management. The research validates the importance of performance methods that are appropriate to a national context, enabling the integration and control of information among stakeholders in a collaborative virtual building model and the data ownership through performance models configured by continuous analysis of the processes, organization and product. This comprehension enables the documentation of effective practices in a database for the design process management setting the model's performance.

KEYWORDS: VDC/BIM Performance analysis, VDC Scorecard, Design process management.

\section{How to cite this article:}

BRITTO S.; SCHEER S.; KAM C.; FISCHER M. O uso do VDC scorecard na validação de métodos para análise de desempenho da gestão do processo de projeto no cenário brasileiro. Gestão e Tecnologia de Projetos, São Paulo, v. 10, n. 2, p. 71-86, jul./dez. 2015

http://dx.doi.org/10.11606/gtp.v10i2.102844

Fonte de financiamento: CAPES - Coordenação de Aperfeiçoamento de Pessoal de Nível Superior $\mathrm{CNPq}$ - Conselho Nacional de Desenvolvimento Científico e Tecnológico Conflito de interesse: Declaram não haver Submetido em: 26 ago. 2015 Aceito em: 1 set. 2015 


\section{INTRODUÇÃO}

Na construção civil, a análise da disseminação da informação entre colaboradores de disciplinas diversas inseridos no processo geral é um coeficiente essencial na configuração da qualidade cíclica do produto, uma vez que a ausência da gestão do desempenho destas influencia na recorrência de problemáticas no planejamento, desenvolvimento, documentação, execução e determinações operacionais da indústria da arquitetura, engenharia, construção e operação (AECO).

A utilização de tecnologias da informação na construção civil, como Virtual Design and Construction (VDC)/Building Information Modeling (BIM), vem se tornando uma importante temática nas pesquisas de boas práticas no setor da arquitetura, engenharia e construção AEC (KUNZ; FISCHER, 2012). Ao sugerir soluções alternativas para melhoria na gestão de processo de projeto, não se deve basear exclusivamente na implementação de novas tecnologias, mas também no direcionamento de esforços para revisão dos processos que contribuirão para o planejamento de melhores práticas, medição e análise de desempenho das mesmas.

A atualização de critérios e métricas para análise de desempenho VDC/BIM existentes no VDC Scorecard tem demonstrado, segundo Kam et al. (2013) e Kam et al. (2014), os benefícios da visualização, integração e automação de tarefas na AECO, em particular, para prever os resultados do projeto e gerenciamento para alcançar o desempenho desejado. À medida que novos conceitos e métodos para melhores práticas na gestão de processo de projeto são desenvolvidos, ainda que para integrar e automatizar estas tarefas, pesquisadores e profissionais devem determinar o valor (ou seja, se o novo método é melhor do que os métodos existentes) desses avanços em projetos reais.

O artigo aborda a contextualização do VDC/BIM no cenário internacional, com enfoque não apenas nos benefícios registrados, mas também na validação de métodos que evidenciem o desempenho da gestão do processo de projeto, como o VDC Scorecard, que possam ser adaptados à realidade do cenário nacional viabilizando estimular a necessidade da documentação da informação para AECO (método, processo e produto) com objetivo de melhores práticas no setor. Trata-se de uma pesquisa descritiva tendo por base pesquisa bibliográfica e documental existentes em bases de dados internacionais e nacionais, bem como alguns resultados de aplicação do método VDC Scorecard.

\section{PANORAMA E DEFINIÇÕES}

A tecnologia da informação não é novidade na indústria da construção, contudo a implementação de plataformas, softwares e sistemas para aplicações específicas têm evoluído ao longo dos anos, tornando-se diversificada a ponto de ser necessário estabelecer critérios e métricas para mensuração de seus benefícios na prática. De acordo com StaubFrench e Fischer (2001), a implementação das tecnologias da informação da construção civil, como Building Information Modeling (BIM) e Virtual Design and Construction (VDC) representam uma nova perspectiva para melhores práticas na AECO e têm sido cada vez mais utilizadas para ampliar a eficiência na gestão de processo de projeto, refletindo em todas as etapas do ciclo de vida da edificação. Entretanto, é importante identificar suas estruturas de conhecimento, dinâmica interna e requisitos para implementação de uma TI, evitando confusão, senso comum, duplicação de esforços, retrabalho.

Segundo Succar (2012), o BIM refere-se a um conjunto de políticas interativas, processos e tecnologias que geram uma metodologia para gerenciar o projeto para indústria da construção e dados essenciais 
em formato digital ao longo do ciclo de vida do edifício. O autor cita a interação entre este conjunto viabilizando a identificação da estrutura de competências para melhor avaliação da maturidade e desempenho BIM.

Para Kassem e Amorim (2015), "o impacto do BIM não se limita às edificações, mas alcança desde a indústria de produtos e materiais, passa pelos projetos e obras de edifícios, estradas e outros tipos de infraestrutura e se prolonga pela manutenção e desmonte ou reuso destas obras. Entretanto, o setor da construção de edifícios pode ser visto como elemento central deste processo de difusão, pois cria demanda para os demais setores e assim sendo, neles orienta a difusão do BIM" (p.19).

Muitos centros de pesquisa reconhecidos internacionalmente, como o Center for Integrated Facility Engineering (CIFE) da Stanford University (2015), na Califórnia, realizam estudos e monitoramentos contínuos em empresas da AECO sobre os benefícios e limitações nos processos de integração multidisciplinar da informação para construção civil com uso do VDC/BIM viabilizando a avaliação da sua maturidade e desempenho.

Segundo Kunz e Fischer (2012), enquanto o BIM tem uma tendência a se agrupar em torno de um modelo de produto e aspectos técnicos de um projeto, o VDC abrange holisticamente a utilização multidisciplinar de modelos de desempenho de Processo, Organização e Produto (POP) e métodos sociais para alcançar os objetivos de negócios, ampliando a eficiência na avaliação do desempenho da gestão do processo de projeto.

De acordo com Kam et al. (2013), desde 2009, mais de 80\% das principais empresas da AECO nos Estados Unidos haviam adotado aplicações BIM. A McGraw Hill Construction (2012) divulgou que sua adoção se intensificou, passando de 28\% em 2007 para 71\% em 2012. O CIFE monitorou o uso multidisciplinar destas aplicações e adoção de métodos sociais, através dos quais os recursos dos aplicativos BIM podem ser aproveitados. No entanto, segundo Kam et al. (2014), a indústria da AECO ainda não efetivou a consolidação de uma estrutura consistente para avaliação do desempenho.

Apesar do potencial do BIM percebido pela indústria, de acordo com Barlish e Sullivan (2012), a maioria das empresas de construção não utiliza uma metodologia formal para avaliar os seus benefícios. Há necessidade de uma metodologia relevante para avaliar os benefícios esperados do BIM em qualquer tipo de projeto, a partir de uma perspectiva de negócios, em conjunto com uma base de dados válida.

Apesar da escassez de metodologias de avaliação, muitos profissionais e pesquisadores têm realizado estudos teóricos e práticos para apoiar a indústria. De acordo com Ho, Kam e Fischer (2009) pelo menos 22 artigos sobre o uso de VDC em projetos individuais têm sido publicados desde 1995, e 23 notáveis diretrizes relacionadas ao VDC direcionadas ao nível da empresa ou da indústria estão disponíveis em base de dados internacionais. Estas iniciativas têm feito alguns progressos no sentido de desenvolver uma metodologia de avaliação VDC/BIM baseada em uma estrutura de conhecimento holístico e adaptável. Segundo Kam et al. (2013), poucas destas metodologias de avaliação são eficazes ou utilizadas pelos profissionais da indústria da AECO, e não são consideradas as deficiências nos processos no que diz respeito à maturidade do desempenho.

\section{METODOLOGIAS PARA AVALIAÇÃO DO DESEMPENHO}

Ferramentas de avaliação são importantes na evolução de boas práticas da indústria. Estas podem ser úteis na qualidade da tomada de decisões e para mensurar os impactos na implementação de novos processos e atualização de processos já implementados. De acordo com Bloom e Van Reenen (2007), boas práticas de gestão que utilizam ferramentas inovadoras para rastrear e monitorar o processo estão relacionadas aos índices mais elevados de produtividade, rentabilidade e crescimento de vendas. 
A definição de métricas em projetos de construção é um desafio na avaliação de mudanças e benefícios. De acordo com Barlish e Sullivan (2012), os termos "Key Performance Indicator" (KPI) e "medição da produtividade" são termos comuns, porém a maioria dos pesquisadores definem com pouca consistência. Os mesmos autores citam modelos como: método da perda de produtividade, análise de milhas medidas, análise da produtividade da linha de base, sistema de modelagem dinâmica, análise de valor agregado, métodos de amostragem e métodos de comparação - são comumente referidos, mas de forma inconsistente utilizados em estudos de caso pouco específicos. Mais comumente, os projetos de construção são medidos através de KPIs; no entanto muitas vezes estes não são uniformes entre os projetos e resultam em confusão em relação a: o que deve ser medido, como deve ser medido, quais são as fontes de mudança, e como avaliar o sucesso ou o fracasso do projeto. Além disso, com esses modelos sugeridos e KPIs, poucos estudos utilizam dados do projeto internos e externos com resultados mensuráveis para validá-los. A produtividade é uma medida popular, mas é baseada em uma quantidade subjetiva, observável.

Segundo Cox, Issa e Ahrens (2003), os KPIs são compilações de medidas de dados usados para avaliar o desempenho de uma operação da construção ou uma tarefa particular. De acordo com levantamento de dados em literatura relacionada à temática, Barlish e Sullivan (2012) evidenciaram a definição de dois tipos de KPIs: qualitativos e quantitativos. Os qualitativos sugeridos pela literatura são: segurança, rotatividade, absenteísmo, e motivação. Em contraste, os exemplos de KPIs quantitativos sugeridos pelos autores são: custo, mão-de-obra, recursos por tempo de execução. Porém, ainda segundo Barlish e Sullivan (2012), estes KPIs não são suficientemente precisos e resultam em excesso de medições subjetivas evidenciando a importância da definição de métricas na medição de desempenho de processos relacionados à $\mathrm{AECO}$.

\section{Métricas BIM}

O uso colaborativo de BIM deve ser mensurável, possibilitando evidenciar melhorias na produtividade e benefícios oriundos da sua implementação. Sem a definição de métricas precisas na avaliação do desempenho das suas aplicações, stakeholders são incapazes de analisar com eficiência os resultados obtidos, sejam estes rentáveis ou que viabilizem a identificação das deficiências.

Segundo Succar, Sher e Williams (2012), métricas de desempenho permitem que as equipes e organizações avaliem as suas próprias competências no uso do BIM e, potencialmente, para avaliar o seu progresso contra a de outros profissionais. Uma estrutura baseada em valores, por exemplo, foi proposta por Barlish e Sullivan (2012), na qual foram analisados os resultados monetários, os fatores organizacionais e o impacto resultante do BIM. Nesta, os pesquisadores evidenciaram a análise de benefícios resultantes da avaliação não apenas dos rendimentos, mas também dos investimentos necessários para consolidação do BIM. A conclusão sobre a totalização dos valores percentuais dos investimentos resultou numa economia do valor total de projeto e construção de $5 \%$. Ou seja, o aumento nos investimentos na etapa de projeto refletiu na qualidade dos processos e, principalmente, na economia dos contratantes.

De acordo com Succar (2010), embora seja importante a definição de métricas e critérios de referência para avaliação do desempenho do uso colaborativo BIM, é necessário que estas, além de precisas, também sejam adaptáveis a diferentes segmentos da indústria. Além disso, conjuntos consistentes de métricas BIM formam as bases de sistemas de certificação formais viabilizando, por exemplo, a seleção de empresas que utilizam a 
plataforma em determinados níveis de maturidade exigidos. O mesmo autor identificou critérios de desempenho que corroboram o aumento da confiabilidade, adotabilidade, e usabilidade para diversos agentes envolvidos no processo. As métricas devem ser:

- Exatas: bem definidas e capazes de medir desempenho com elevados níveis de precisão;

- Aplicáveis: capazes de serem utilizadas por todas as partes interessadas em todas as fases do ciclo de vida do projeto;

- Atingíveis: realizáveis, se as ações definidas são implementadas como determinadas;

- Consistentes: rendimento de resultados similares quando conduzida por diferentes avaliadores;

- Cumulativas: definidas como progressões lógicas, nas quais as entregas a partir de um ato são pré-requisitos para outro;

- Flexíveis: capazes de serem aplicadas a diversos segmentos, escalas organizacionais e suas subdivisões;

- Neutras: não favorecem apenas um interesse;

- Específicas: direcionada aos requisitos específicos da indústria da construção;

- Universais: aplicáveis igualmente em todos os segmentos;

- Úteis: intuitivas e capazes de serem facilmente empregadas para avaliar o desempenho BIM.

Segundo Succar, Sher e Williams (2013) há um número crescente de métricas de maturidade BIM para avaliar o desempenho de indivíduos, organizações e projetos. Destas, apenas algumas métricas podem ser aplicadas para medir e comparar a maturidade BIM entre países. No mesmo estudo, os pesquisadores propuseram três métricas qualitativas para medir a adoção BIM e maturidade nos países analisados (Austrália, EUA e Reino Unido). Essas métricas abordaram a importância da disponibilidade, distribuição e relevância de publicações notáveis sobre BIM (Noteworthy BIM Publications - NBP) em diversos mercados.

Contudo, segundo Scheer (2015), é importante:

- Conhecer o perfil do elemento a ser avaliado (individual/profissional, empresa, governo, região/país), a fim de definir KPIs corretamente;

- Compreender antecipadamente as consequências de uma escolha KPI ineficiente, se preparando para revertê-lo em ações benéficas para a pesquisa e para os indivíduos pesquisados/organizações;

- Saber sobre as regras e regulamentos específicos para cada cidade, estado, região ou país, bem como as práticas atuais e "vencedoras", políticas internas e ao tipo específico de negócio (que tipo de empresa de projeto/construção: real state, residencial, escritório, industrial, comercial);

\section{Maturidade BIM}

Segundo Succar (2009), a maturidade BIM pode ser caracterizada através da qualidade, repetibilidade e grau de excelência dentro de uma competência do uso colaborativo de BIM. Ainda segundo o autor, "maturidade" denota a extensão dessa competência na realização de uma tarefa ou prestação de um serviço com o BIM. Esta corresponde à progressão dos níveis iniciais a níveis mais elevados de maturidade, indicando respectivamente:

- maior controle, resultante de menor variação entre as metas de desempenho e os resultados reais;

- maior previsibilidade para alcançar objetivos, custo, tempo de desempenho;

- maior eficácia para alcançar objetivos definidos e mais confiabilidade para planejamentos futuros.

O conceito de maturidade BIM em Succar, Sher e Williams (2012) foi adotado a partir do modelo de maturidade da capacidade (CMM). Este 
foi concebido em função da necessidade de melhoria de processos para avaliação dos fluxos de entrega de projetos de software pelos fornecedores do governo e posteriormente para benefícios do Departamento de Defesa dos EUA.

De acordo com Goetze (2014), existem muitas tentativas para mensurar os níveis de maturidade BIM. Os modelos utilizados são geralmente divididos em duas categorias, medindo a eficiência e eficácia BIM no âmbito de projetos de construção e entre organizações. $\mathrm{O}$ autor cita três dos modelos que considera mais relevantes:

- National Institute of Building Sciences (2007) - National BIM Standard's Interactive Capability Maturity Model (ICMM): o modelo ICMM define onze características que configuram a pontuação mínima. Estas características são relacionadas aos níveis de maturidade resultando em uma matriz que é ponderada de acordo com os valores encontrados e convertidos em uma pontuação final concedida ao projeto de construção;

- Succar, Sher e Williams (2012) - Framework and BIM Maturity Index (BIMMI): desenvolvido através da análise e compilação de modelos de maturidade dentro de diversos segmentos da indústria. Destinase a reproduzir os indicadores de gestão da qualidade, requisitos de implementação e avaliação do desempenho BIM. Succar, Sher e Williams (2012) desenvolve seu modelo baseado na análise em três campos interligados: processos, tecnologia e política;

- - BIS (2011): iBIM Maturity Model; o modelo iBIM foi elaborado para garantir articulação clara das diretrizes e normatizações e como estas podem ser aplicadas a projetos e contratos na indústria da construção no Reino Unido. O modelo identifica as metas específicas de competência, cobrindo tecnologia, normas, classificações e contrato. Estas metas caracterizam a definição de maturidade representada em três níveis no modelo.

É importante mencionar que existem outros esforços relacionados à maturidade em BIM como os citados por Kassem et al. (2013).

\section{VDC SCORECARD}

Segundo Kam et al. (2013), a apropriação de dados coletados a partir dos modelos de desempenho configurados por meio da análise contínua dos procedimentos a serem executados, organização e produto possibilitam a documentação de práticas eficientes para gestão de processos de projeto, configurando o desempenho do modelo. Estes desempenhos são avaliados mediante utilização dos valores percentuais abordados nas quatro (04) áreas que compõe a metodologia de medição e gestão do desempenho do VDC/BIM. Estas áreas são definidas por meio do VDC Scorecard, compostas por métricas de planejamento, adoção, tecnologia e desempenho que configuram a pontuação geral medida em uma escala percentual que reflete o desempenho do projeto em relação à prática da indústria.

O VDC Scorecard é um método de avaliação da maturidade do uso colaborativo VDC/BIM nas práticas da indústria AECO. Esta maturidade é evidenciada por atribuição de valores que configuram a pontuação geral do desempenho das unidades estudadas no âmbito da definição de métricas qualitativas e quantitativas compostas pela análise estatística da evolução contínua das práticas na indústria. Estes valores de maturidade são hierarquizados em níveis do VDC Scorecard: a prática convencional (0\%-25\%), prática típica (25\%-50\%), prática avançada (50\%-75\%), melhores práticas (75\%90\%), e práticas inovadoras (90\%-100\%). A Figura 1 abaixo representa esta hierarquização com o quadro de diagnóstico do método. 


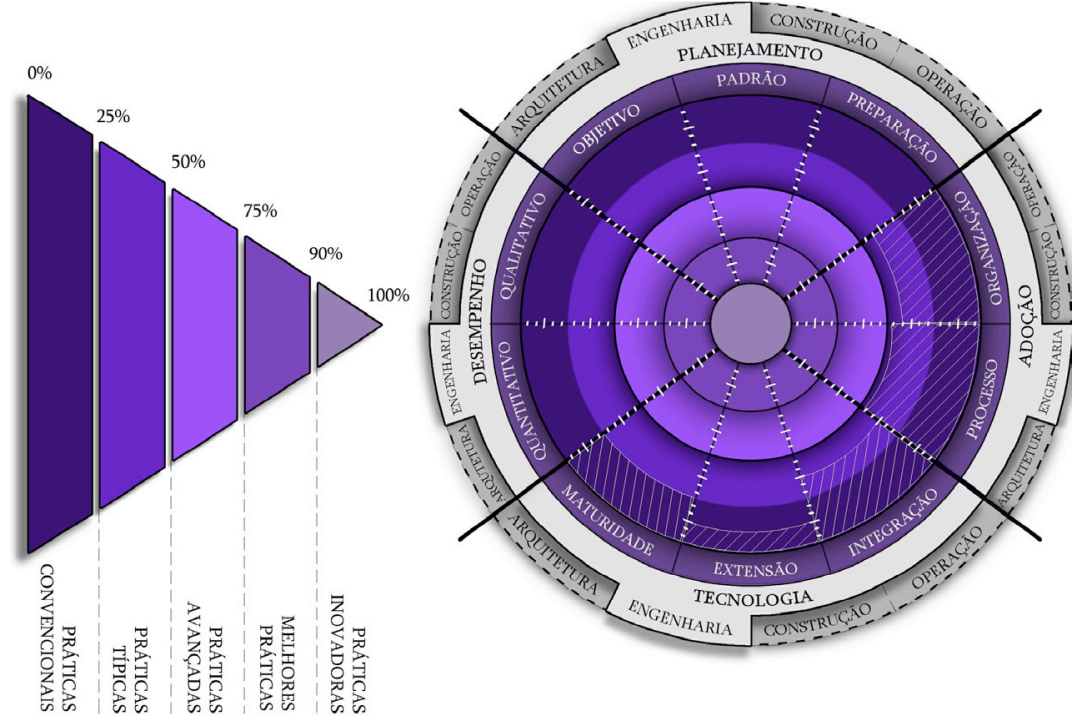

O VDC Scorecard utiliza uma escala percentual que é composta por cinco níveis de desempenho para cada uma das 56 métricas, e complementada com uma avaliação da segurança na pontuação. Os percentuais e as camadas são definidos por meio de observações de práticas da indústria. O método é composto por quatro áreas, 10 divisões, e 56 métricas, evidenciando o nível de confiança avaliado por sete fatores para indicar a precisão na pontuação global. Essas quatro áreas são:

- A área de planejamento, que abrange a criação de objetivos e normas, bem como a disponibilidade de recursos tecnológicos e fiscais que promovam os objetivos de negócio dos projetos;

- A área de desempenho, na qual a precisão e qualidade de informações obtidas sobre os objetivos alcançados são mensurados pela análise quantitativa e qualitativa;

- A área de adoção, que avalia os aspectos organizacionais e processuais de métodos sociais para adotar a tecnologia;

- A área de tecnologia, que avalia os modelos de produto, organização e processos implementados em cinco níveis de maturidade;

A inclusão de medição de nível de confiança fornece uma avaliação mais holística, informando aos usuários a confiabilidade da avaliação. O nível de confiança indica a precisão das medições, considerando as fontes, a conformidade de entrada e frequência das avaliações. A Figura 2 representa a estruturação do método, evidenciando o valor referente à pontuação final avaliada.

A pontuação geral do VDC Scorecard é uma medida criada usando média ponderada das pontuações por área correspondente ao VDC Scorecard para quantificar o desempenho geral de um projeto. A pontuação por área (04) é resultado da média ponderada de cada dimensão (10). E cada dimensão calculada com média ponderada para cada métrica (56). Esta pontuação viabiliza a classificação e adoção de estratégias para solução de problemas identificados nos setores que compõem estas áreas. Estas deficiências detectadas são avaliadas com base no planejamento de atividades para ações de melhoria e consequentemente são desenvolvidas recomendações para solução dos problemas detectados.

Para determinar maior confiança no resultado da pesquisa, os casos estudados são analisados conforme os sete fatores que classificam o nível de confiança do levantamento:
Figura 1. Nível de maturidade e diagnóstico VDC

Scorecard. Fonte: STANFORD UNIVERSITY/CIFE, adaptado pelos autores (2015). 


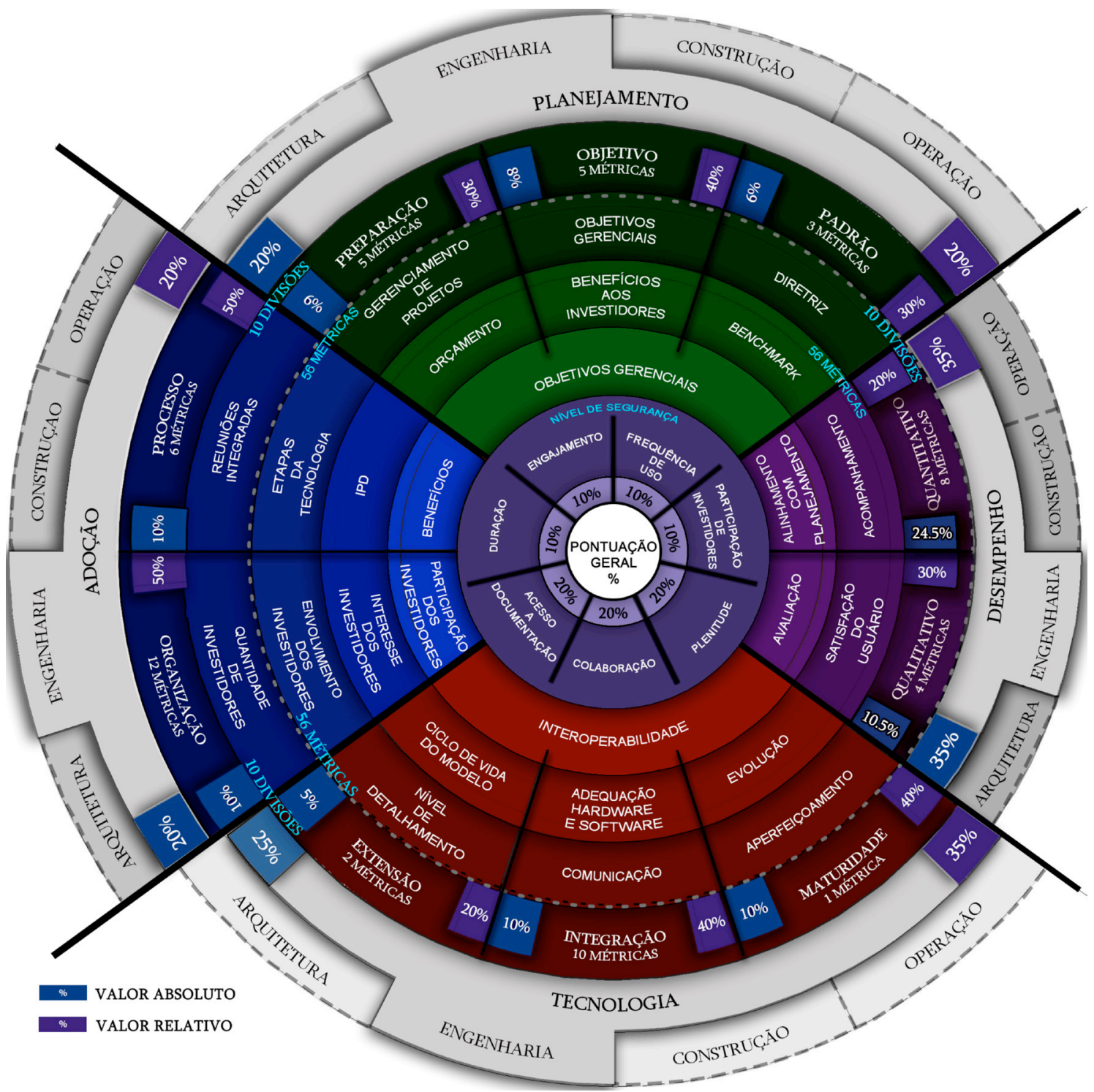

Figura 2. Estrutura VDC

Scorecard. Fonte: STANFORD UNIVERSITY/CIFE, adaptado pelos autores (2015)
- Colaboração: o conhecimento de colaboradores com maior experiência no processo eleva o nível de confiança da pesquisa;

- Plenitude: disponibilidade dos envolvidos em participação e conclusão de todas as etapas da pesquisa;

- Duração: quanto maior o tempo disponibilizado para desenvolvimento da coleta de dados, maior o nível de confiança;

- Acesso à documentação: a pontuação referente à confiança da pesquisa aumenta com a disponibilização de documentos relacionados aos questionamentos;

- Engajamento: quanto mais próximo da etapa de conclusão e entrega do projeto, maior o nível de confiança em comparação a um projeto em estágio inicial;

- Participação de investidores: quanto maior for o nível de participação dos envolvidos, maior o nível de confiança da pesquisa;

- Frequência de uso: quanto maior a frequência de coleta de dados, maior é a precisão dos resultados e, consequentemente, mais elevado seu nível de confiança; 
De acordo com Ho, Kam e Fischer (2009), o desenvolvimento de uma metodologia de avaliação VDC/BIM não influencia apenas na qualidade da informação, mas também no desenvolvimento de base de dados gerenciais para melhoria constante na indústria trazendo também oportunidades para novas pesquisas e críticas construtivas que podem basear-se em uma base de conhecimento sólida e bem fundamentada. Esta base viabiliza a retroalimentação do conhecimento entre as universidades, as organizações públicas e privadas e diversos segmentos da indústria.

De acordo com Kam et al. (2014), os critérios para estruturação do método foram desenvolvidos considerando os seguintes aspectos:

- Holístico: abrangendo a avaliação do desempenho de aspectos técnicos do projeto com informações obtidas por colaboração social;

- Quantificável: uma estrutura de avaliação requer medidas objetivas e quantificáveis que possam ser utilizadas para monitorar e acompanhar o progresso do projeto e a maturidade VDC;

- Prático: o quadro de avaliação tem de ser acessível aos profissionais da AECO, pois retrata o desempenho do VDC/BIM de um projeto sobre uma série de medidas em relação aos padrões da indústria;

- Adaptável: a estrutura de avaliação deve ser capaz de se adaptar à diversidade e evolução das práticas no setor da AECO;

Pesquisadores e profissionais da AECO têm produzido uma série de orientações e documentos de pesquisa sobre o uso do VDC/BIM que influenciaram o desenvolvimento do VDC Scorecard (KAM et al. 2014). Embora alguns destes documentos possuam conteúdos abrangentes que cobrem muitos aspectos POP, mantendo uso prático na indústria, cada um deles contribuiu em sua respectiva área de conhecimento e prática. Por isso, é importante construir considerando as suas contribuições e pontos fortes, bem como suas limitações e fraquezas no contexto dos objetivos. Na subseção seguinte serão evidenciados alguns resultados de casos estudados.

\section{Benefícios registrados}

Segundo estudos realizados pelo grupo de pesquisa do CIFE, até o final de 2012 foram analisados 108 projetos da AECO. Estes estão distribuídos em 13 países da América do Norte, Europa, Ásia e 15 estados dos EUA, e foram projetados e construídos por empresas da AECO em várias regiões. Os resultados e pontuações destes projetos foram analisados utilizando o VDC Scorecard, a fim de estabelecer correlações entre as práticas, bem como a avaliação de desempenho VDC/BIM na indústria. De acordo com Kam et al. (2013), dos 108 projetos estudados, 2 estavam abaixo de $25 \%$ e foram classificados como "Prática convencional"; 49, entre $25 \%$ e $50 \%$, sendo caracterizados como "Prática típica"; 52 projetos foram pontuados entre $50 \%$ e $75 \%$ e foram considerados "Práticas avançadas"; 5, entre 75\% e 90\%, considerados "Melhores práticas"; e nenhum projeto foi pontuado entre $90 \%$ e $100 \%$, o que os caracterizaria como "Prática inovadora". Estes estudos evidenciaram maior pontuação para os projetos localizados nos EUA em comparação com os de outros países. Os autores relacionam este resultado ao maior investimento pelos stakeholders na implementação do VDC/BIM no setor da construção americana, enfatizando a importância da participação e maior conhecimento destes na adoção de melhores práticas na indústria da AECO. Um exemplo desta observação pode ser analisado na avaliação da utilização do VDC/BIM no cenário da AECO nacional, em estudos realizados em projetos brasileiros.

No período de janeiro a março de 2015, em disciplina ofertada pelo CIFE, foi desenvolvido um estudo de aplicação do VDC Scorecard em seis projetos brasileiros. Dois desses projetos tiveram destaque em função das pontuações registradas, evidenciando o perfil atual do cenário brasileiro na adoção do VDC/BIM. Trata-se de projetos de edificações residenciais e empresariais situados na cidade de Curitiba/PR que foram analisados no 
âmbito das quatro áreas que compõem o VDC Scorecard. Em função da diferença no cenário da construção civil brasileira, da execução de ambos os casos ainda estar em andamento e da quantidade de respondentes, as pontuações referentes ao nível de confiança das avaliações foram baixas (16\%). Entretanto, a pontuação global registrada pela análise do VDC Scorecard, evidenciou resultados próximos ao nível de "práticas avançadas" (48 e 49\%) devido aos valores registrados na área referente à análise tecnológica (49 e 59\%) em função da maior integração da informação no modelo desenvolvido e consequentemente na avaliação qualitativa e quantitativa do seu desempenho (55 e 50\%). Nessa análise, as deficiências ainda existentes pelo pouco aproveitamento de informações extraídas do modelo para documentação, a necessidade de maior variedade e melhor análise de projetos complementares (estrutural, elétrico, hidrossanitário, HVAC), junto com o pouco detalhamento destes sistemas, correspondem à insuficiência na obtenção da pontuação máxima. Abaixo são evidenciadas as principais considerações que influenciaram na pontuação nestas duas áreas:

1) Tecnologia

- Maior integração da informação no modelo, possibilitando melhor análise no 4D, 5D e detecção de interferências (clash detection). Sem perdas ou retrabalhos após transferência (IFC) da produção entre os envolvidos;

- Necessidade de ênfase no uso do modelo como ferramenta para melhor extração e documentação da informação multidisciplinar, não apenas como meio para detecção de interferências físicas na modelagem;

- Utilização do modelo principal (arquitetônico) para visualização e comunicação entre os projetistas. Necessidade de melhor aproveitamento do modelo BIM na elaboração de documentos que viabilizem melhor a comunicação entre os envolvidos;

- Maior detalhamento e aproveitamento de informações para melhor desenvolvimento de projetos complementares no modelo BIM. Incluir informações de sistemas (HVAC, mecânico, elétrico, equipamentos) no modelo, viabilizando maior precisão na detecção de interferências;

2) Desempenho

- Uma das análises foi caracterizada pelo estágio de desenvolvimento dos processos. Os projetos nesta análise correspondem a níveis de informação referentes à fase de entrega do produto resultante da implementação parcial da plataforma. O mesmo está em fase de execução da obra e atingiram o nível de satisfação do cliente. Contudo, ainda são necessárias melhorias na comunicação entre os projetistas, visando eficiência na solução de incompatibilidades projetuais identificadas no modelo colaborativo;

- Apesar de um dos estudos analisados ainda não ter tido retorno em relação ao investimento com a implementação gradativa da plataforma, o nível de satisfação por parte dos stakeholders foi elevado em função do esforço na comunicação, viabilizando maior participação entre as partes envolvidas. Ainda é necessário haver maior participação dos investidores no processo;

- Ambos os estudos buscaram máximo alinhamento entre os objetivos definidos pelos investidores em relação ao custo e prazo com a utilização da plataforma, e até a realização da coleta de dados, o nível de satisfação do cliente correspondeu aos objetivos esperados.

Além das considerações citadas referentes às duas áreas que obtiveram maior pontuação na análise dos estudos, foram coletadas informações que diagnosticaram os pontos fortes, deficiências e possibilidades de melhoria nas duas demais áreas com scores inferiores, classificadas como "práticas 
típicas”. A disparidade entre os maiores valores obtida entre a análise tecnológica (59\%, "práticas avançadas") e menores valores relacionados à análise do planejamento (33\%, "práticas típicas") influenciaram na pontuação global, refletindo na caracterização do perfil dos casos estudados. Ainda que sejam necessários estudos mais criteriosos em decorrência dos diversos fatores e possíveis métricas a serem consideradas ou não aplicáveis a nível nacional, é perceptível a deficiência nas práticas correspondentes ao planejamento de projeto no cenário brasileiro. O mesmo influencia na adoção de novos conceitos, estratégias e eficiência no monitoramento e tomada de decisões essenciais para revisão de processos e qualidade final do produto. O planejamento de execução para implementação eficiente da plataforma é fundamental para maior controle e qualidade no gerenciamento do processo. Considerando que a retroalimentação da informação é essencial para definição de melhores práticas no uso colaborativo VDC/BIM, torna-se imprescindível direcionamento de esforços para analisar o planejamento e desempenho das mesmas, evitando ciclo vicioso de práticas obsoletas ou pouco produtivas em relação ao potencial da plataforma.

Tendo o desempenho dos casos estudados atingido um score responsável pela atribuição de valores resultantes da análise tecnológica classificada como próxima às "práticas avançadas", é necessária a revisão de algumas diretrizes do planejamento e, consequentemente, do processo de implementação. A pontuação relacionada ao planejamento e adoção do VDC/BIM foi baixa em comparação às áreas citadas anteriormente. As razões variam desde inexistência de incentivos contratuais até inexistência de planejamento de execução para adoção e revisão de processos em função da implementação da plataforma, inviabilizando melhores práticas e maior eficiência no produto final.

As principais considerações que influenciaram na pontuação nestas áreas foram:

1) Planejamento

- Inexistência de planejamento para execução da implementação da plataforma. Em ambos os casos estudados, o uso do BIM foi caracterizado por não seguir diretrizes estabelecidas para maior controle na implementação dos processos e consequentemente formalização de melhores práticas para a empresa;

- Inexistência de controle de informações que contribuam para a formalização de banco de dados gerenciais de melhores práticas para uso do BIM no Brasil;

- Objetivos gerenciais definidos, porém inexistência de diretrizes básicas para alinhamento de informações com a evolução dos processos e obtenção dos resultados desejados a curto, médio e longo prazo;

- Ambos os casos estudados obtiveram razoável avaliação em relação à capacitação e treinamento dos profissionais que compõem a equipe BIM;

- Sem aplicações ou utilização do BIM para operação e manutenção (O\&M);

2) Adoção

- Deficiência no compartilhamento formal do conhecimento e informação entre agentes;

- Inclusão de responsabilidades e incentivos contratuais. Revisão de atribuições entre os envolvidos, evitando sobrecarga de funções, ociosidade e absenteísmo ("quem”, “como", "quando" e "nível de desenvolvimento");

- Inexistência de análises e otimizações baseadas no modelo para projetos complementares;

- Necessidade de ênfase em treinamentos e capacitação de profissionais responsáveis pelos projetos complementares (estrutural, elétrico, hidrossanitário, HVAC, mecânico); 
Figura 3. Resultados da aplicação do VDC Scorecard - Projeto 2. Fonte: Strategic Building Innovation (2015) http://www.sbi.international.

Figura 4. Comparação de resultados da aplicação para os seis projetos. Fonte: Strategic Building Innovation (2015) http://www.sbi.international.
- Necessidade de criação de biblioteca para armazenamento de detalhamentos e objetos parametrizados para utilizações futuras;

- Necessidade de ênfase em treinamentos e capacitação de profissionais responsáveis pelo desenvolvimento 4D. Necessária maior participação dos stakeholders para maior compreensão do modelo.

Na Figura 3 é representado um gráfico com resultados obtidos a partir da utilização da versão on-line do VDC Scorecard para um dos projetos estudados (http://www.sbi.international).

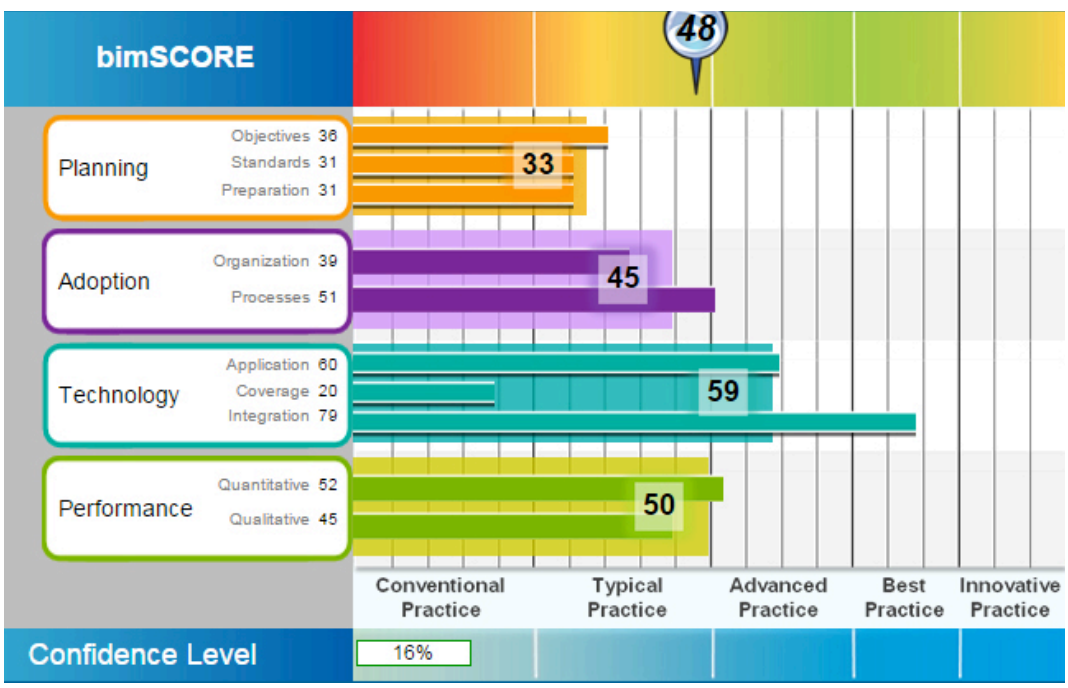

Na Figura 4 está representado um gráfico de comparação (benchmarking) entre os seis projetos brasileiros estudados.

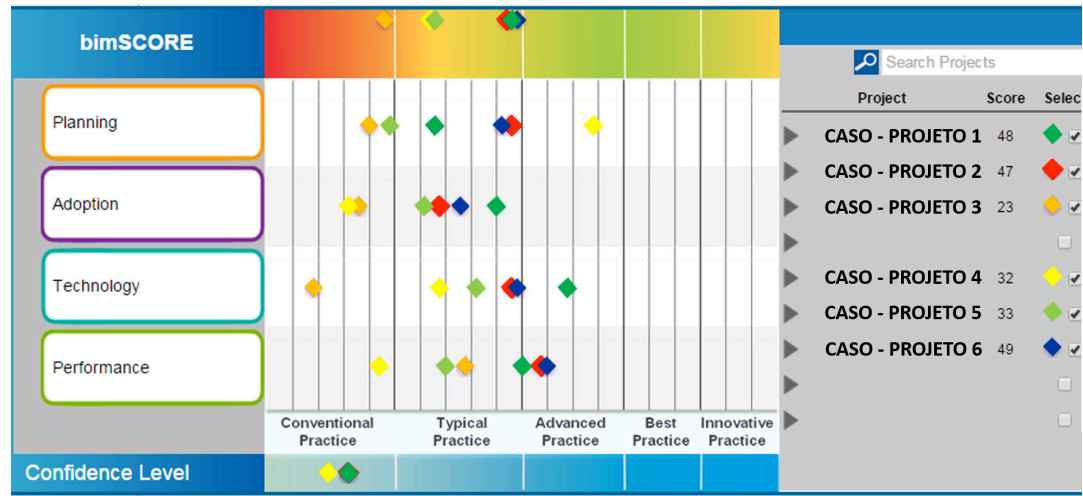

Considerando resultados em relação ao investimento pelos stakeholders na implementação do VDC/BIM no setor da construção dos EUA, torna-se necessário fomentar maior participação e conhecimento dos envolvidos na adoção de melhores práticas na indústria da AECO brasileira. Estas práticas necessitam ser avaliadas para definição da maturidade dos processos e aproximação de resultados que viabilizem qualidade na tomada de decisões e, sobretudo, documentação de banco de dados gerenciais para práticas eficientes.

Há ainda insegurança e descrença (menor que em anos anteriores) perante riscos no investimento (principalmente diante do atual cenário político-econômico nacional) para adoção de inovações na gestão de processos para construção civil. Ainda que seja fundamental considerar as adversidades relacionadas ao conservadorismo na gestão, ausência de 
estratégias para implementação, mensuração de resultados, capacitação de agentes na TI, com o crescente, porém árduo, processo de padronização para troca de dados (interoperabilidade), é inevitável considerar a adoção da plataforma. A mesma, quando implementada de forma eficiente com revisões dos processos organizacionais, tem potencial para proporcionar melhor qualidade nos processos em ambiente colaborativo multidisciplinar da AECO a curto, médio e longo prazo. Contudo, existe interesse crescente, porém ainda lento, por parte dos stakeholders na adoção e utilização do VDC/BIM no setor da construção civil brasileira em função dos benefícios registrados pela utilização da plataforma. A subseção seguinte abordará considerações, resultados e perspectivas deste progresso no cenário brasileiro.

\section{Cenário nacional}

Kassem e Amorim (2015) apontam o setor da construção brasileira como um dos maiores globalmente, sendo responsável por 2\% do total. Em um cenário globalizado, o contexto desejável de benefícios tem incluído melhorias na eficiência e sustentabilidade da construção e operação dos empreendimentos, bem como na previsibilidade de retorno e valor dos investimentos e também incremento de exportação com crescimento econômico.

De acordo com estudos desenvolvidos por Goetze (2014), a indústria brasileira da construção está gradualmente assimilando as considerações provenientes da implementação do BIM. A aplicação correta do BIM implica na revisão de negócios, modifica relações na indústria desde empreiteiros até fabricantes, dos clientes aos projetistas. Além disso, as organizações envolvidas com o BIM precisam de modificações internas, e sua eficiência e eficácia se baseiam na confiança entre os membros diferentes, as equipes e stakeholders. O processo de adoção BIM deve ser cuidadosamente nutrido e planejado para que a indústria possa explorar todo o seu potencial.

Benefícios citados por profissionais da AECO brasileira em entrevistas desenvolvidas por Goetze (2014) evidenciaram o fluxo emergente de interessados na implementação da plataforma. Algumas organizações realizaram estudos nos quais contratantes avaliaram os custos de um projeto de pequeno porte e concluíram que o uso de BIM teria economizado por volta de $15 \%$ a $20 \%$ dos custos totais, enquanto um cliente mediu a otimização de custos de 6,7\% em um grande contrato. Entretanto, 100\% dos entrevistados tinham um elevado número de detecções de incompatibilidade (clash detection) em todos os projetos graças ao BIM. No entanto, como os subcontratantes do projeto ainda não começaram a usar BIM, os custos de projetos subiram, segundo o autor. Os entrevistados têm evidenciado a transferência dos custos da fase de construção para a fase de concepção do projeto. Esta evolução contribui para análise de melhores práticas por meio do monitoramento da maturidade do VDC/BIM de forma constante.

De acordo com McGraw Hill Construction (2014), o cenário brasileiro possui grandes perspectivas em relação à utilização do VDC/BIM. O nível de interesse e investimento por parte de construtoras nacionais tem aumentado em função dos benefícios registrados de maior Return On Investiment (ROI - Rendimento sobre Investimento). Segundo este relatório, o Brasil possui um valor percentual progressivo de $36 \%$ de ROI em VDC/BIM por conta das construtoras nacionais, próximo de países como Alemanha, Canadá, França e Japão. A razão do interesse emergente, segundo resultados divulgados pela Smart Market Report (2014), são maiores qualidade dos projetos e processos; melhoria da produtividade e melhor comunicação e compreensão por parte de visualização tridimensional multidisciplinar. Apesar do tempo de experiência e níveis de maturidade dos processos ainda em estágios iniciais (70 e 55\% em níveis primários, respectivamente), as construtoras nacionais representam uma porção emergente na utilização do VDC/BIM, com níveis 
crescentes de expertise dos profissionais envolvidos nos processos (entre moderado e avançado).

No âmbito do programa Diálogos Setoriais União Europeia - Brasil, Kassem e Amorim (2015) conduziram um estudo sobre a adoção de BIM no Brasil e em cinco países da Europa (Reino Unido, França, Holanda, Finlândia e Noruega). Na iniciativa do Ministério do Desenvolvimento da Indústria e Comércio Exterior (MDIC) e do Ministério do Planejamento, Orçamento e Gestão do Brasil, e com apoio da FIESP/DECONCIC para o grupo de trabalho, os consultores desenvolveram um conjunto de recomendações e conclusões sobre a difusão de BIM no Brasil. As recomendações estão divididas em nove áreas, incluindo: estratégias, objetivos e estágios; protocolos e guias; atores líderes; resultados padronizados e bibliotecas de componentes digitais; arcabouço regulatório; medidas e otimização; educação e treinamento; infraestrutura e tecnologia; e viabilidade econômica e pesquisa de iniciativas de construção.

Assim como o Smart Market Report (2014), esse relatório produzido no âmbito dos Diálogos Setoriais União Europeia-Brasil aponta que a adoção de BIM é bastante avançada entre construtoras de médio e grande porte. Todavia, o uso no Brasil é mais voltado ao controle de custos da fase de construção e menos na colaboração entre os envolvidos no empreendimento. Isto representa um uso reverso do BIM quando comparado àquele verificado em outros países como o Reino Unido, França, Estados Unidos da América e Alemanha.

Como postulado por Kassem e Amorim (2015), “o estabelecimento de um sistema de medição do desempenho do BIM, seja individual (competências) ou para organizações (capacidade e maturidade), é o primeiro passo para o caminho de desenvolvimento de um sistema de certificação e auditoria no futuro". Não foi encontrado por estes autores nenhum esquema bem estabelecido para medição e otimização de desempenho VDC/BIM.

\section{CONCLUSÃO}

A avaliação do desempenho em VDC/BIM é essencial na adoção de melhores práticas no setor da AECO nacional. Por se tratar de um método de avaliação holístico e adaptável baseado em evidências, o VDC Scorecard possui, na sua concepção, métricas que tendem a ser aproveitadas para o cenário brasileiro. Esta apropriação deve estar de acordo com a melhoria e atualização dos processos em função do lento, porém contínuo progresso da integração da informação na AECO nacional com o uso do VDC/BIM.

A atualização contínua do processo avaliativo e validação do VDC Scorecard fundamentam a correlação entre as métricas individuais e avaliação da pontuação geral. Este processo cria um ciclo de feedback positivo, em que o VDC Scorecard serve como método de avaliação para os profissionais da AECO, e seus dados podem ser utilizados para melhorar suas práticas no setor. Este ciclo de feedback positivo proporciona a consolidação de uma base de dados para melhores práticas no setor da AECO. Contudo, é imprescindível a adaptação e avaliação destes processos à realidade do cenário brasileiro, uma vez que eles são caracterizados pela maioria emergente na implementação do VDC/BIM, porém com considerável lentidão em relação aos processos convencionais da indústria nacional.

\section{AGRADECIMENTOS}

À CAPES, junto ao Programa de Pós-Graduação em Engenharia de Construção (PPGECC) da UFPR e ao CNPq (Programa Ciência sem Fronteiras) pela concessão de bolsas. À colaboração dos professores Martin Fischer e 
Calvin Kam e seus alunos na parceria com o Center for Integrated Facility Engineering (CIFE) da Stanford University.

\section{REFERÊNCIAS}

BARLISH, K.; SULLIVAN, K. How to measure the benefits of BIM - a case study approach. Automation in Construction, v. 24, p. 149-159, jul. 2012. DOl: 10.1016/j. autcon.2012.02.008.

BUSINESS INNOVATION AND SKILLS. A report for the Government Construction Client Group, Building Information Modelling (BIM) working party strategy, 2011. Department for Business Innovation \& Skills (BIS). Disponivel em: <http://www. cita.ie/ images/assets/uk\%20bim\%20 strategy\%20(summary).pdf>. Acesso em: 2 maio 2015

BLOOM, N.; VAN REENEEN, J. Measuring and explaining management practices across firms and countries. Quarterly Journal of Economics, Cambridge, v. 122, n. 4, p. 1351-1408, nov. 2007.

COX, R.; ISSA, R.; AHRENS, D. Management's perception of key performance indicators for construction. Journal of Construction Engineering and Management, Blacksburg, v. 129, n. 2, p. 142-151, abr. 2003. DOI: http://dx.doi.org/10.1061/(ASCE)07339364(2003)129:2(142).

GOETZE, G. Building information modelling: maturity and effects on the construction phase of a project in Brazil. 2014. 108 pp. Dissertação (Mestrado) - University College London, School of Construction and Project Management, Londres, 2014.

HO, P.; KAM, C.; FISCHER, M. Prospective validation of virtual design and construction methods: framework, application, and implementation guidelines. CIFE Working Paper \#WP123, Center for Integrated Facility Engineering (CIFE), Department of Civil and Environmental Engineering, Stanford University. Stanford, dez. 2009, p.1-42. Disponível em: < http://cife.stanford.edu/ sites/default/files/WP123.pdf>. Acesso em: 22 out. 2015

KAM, C.; MCKINNEY, B.; XIAO, Y.; SENARATNA, D. The VDC Scorecard: evaluation of AEC projects and industry trends. CIFE Working Paper \#WP136, Center for Integrated Facility Engineering, Dept. of Civil and Environmental Engineering, Stanford University. Stanford, out. 2013, p.1-31. Disponível em: <http://cife.stanford.edu/sites/default/ files/WP136.pdf>. Acesso em: 22 out. 2015.
KAM, C.; MCKINNEY, B.; XIAO, Y.; SENARATNA, D. The VDC Scorecard: Formulation and Validation, CIFE Working Paper \#WP135 v2, Center for Integrated Facility Engineering, Dept. of Civil and Environmental Engineering, Stanford University. Stanford, out. 2013. Revisado jan. 2014. p. 1-40. Disponível em: <http://cife. stanford.edu/sites/default/files/WP135v2. pdf>. Acesso em: 22 out. 2015.

KASSEM, M.; AMORIM, S. R. L. BIM Building Information Modeling no Brasil e na União Europeia. Brasília: Ministério do Planejamento, Orçamento e Gestão, 2015. Disponível em: <http://sectordialogues.org/ sites/default/files/acoes/documentos/bim. pdf>. Acesso em: 29 jun. 2015.

KASSEM, M.; SUCCAR, B.; DAWOOD, N. A proposed approach to comparing the BIM maturity of countries. In: INTERNATIONAL CONFERENCE ON APPLICATIONS OF IT IN THE AEC INDUSTRY, 30., 2013, Pequim. Proceedings of the CIB W78 2013... Pequim: CIB W78, 2013. p. 1-11.

KHANZODE, A.; REED, D.; FISCHER, $M$. Benefits and lessons learned of implementing building virtual design and construction (VDC) technologies for coordination of mechanical, electrical, and plumbing (MEP) systems on a large healthcare project. Journal of Information Technology in Construction, v. 13, p. 324342, jun. 2008. Edição especial.

KUNZ, J.; FISCHER, M. Virtual design and construction: themes, case studies and implementation suggestions. CIFE Working Paper \# 097, Center for Integrated Facility Engineering (CIFE), Dept. of Civil and Environmental Engineering, Stanford University. jun. 2005. Revisado jan.2012. p.1-50. Disponivel em: <http://cife.stanford. edu/sites/default/files/WP097_0.pdf>. Acesso em: 22 out. 2015.

MCGRAW-HILL Construction. The Business value of BIM. Nova York: Smart Market Report, 2012.

MCGRAW-HILL Construction. The Business value of BIM for construction in Major Global Markets. Nova York: Smart Market Report, 2014.

NATIONAL INSTITUTE OF BUILDING SCIENCES. National Building Information Modeling Standard. Washington: National Insitute of Building Sciences, 2007. Disponível em: <http://www.wbdg.org/ 
pdfs/NBIMSv1_p1.pdf>. Acesso em: 2 maio 2015.

SCHEER, S. Some thoughts about the "points of departure". Notes for CEE 112/212B. Spring 2015 Industry Applications of Virtual Design \& Construction at Stanford University, 2015. No prelo.

SMART MARKET REPORT. The Business value of BIM. Nova York: McGraw-Hill Construction, 2012.

SMART MARKET REPORT. The Business value of BIM for construction in Major Global Markets. Nova York: McGraw-Hill Construction, 2014.

STANFORD UNIVERSITY. Center for integrated facility engineering. VDC and BIM Scorecard. (Homepage). Disponível em: <http://vdcscorecard.stanford.edu>. Acesso em: 30 jun. 2015.

STAUB-FRENCH, S.; FISCHER, M. Industrial Case Study of Electronic Design, Cost, and Schedule Integration. CIFE Technical Report \#122, Center for Integrated Facility Engineering (CIFE), Dept. of Civil and Environmental Engineering, Stanford University. Stanford, jan. 2001. p.1-125. Disponível em: <http://cife. stanford.edu/sites/default/files/TR122.pdf>. Acesso em: 22 out. de 2015.

SUCCAR, B. Building information modelling framework: a research and delivery foundation for industry stakeholders.
Automation in Construction, v. 18, n. 3, p. 357-375, 2009. Disponivel em:<http://www. researchgate.net/publication/222267027_ Building_Information_Modelling_ framework_A_research_and_delivery_ foundation_for_industry_stakeholers $>$. Acesso em: 1 out. 2015. DOl:10.1016/j. autcon.2008.10.003.

SUCCAR, B. The five components of BIM performance measurement. In: CIB WORLD BUILDING CONGRESS, 18., 2010, Salford. Proceedings... Salford: CIB, 2010. p. 1-14.

SUCCAR, B.; SHER, W.; WILLIAMS, A. Measuring BIM performance: five metrics. Architectural Engineering and Design Management, v. 8, n. 2, p. 120-142, 2012. Disponível em: <http:// dx.doi.org/10.1080/17452007.2012.65 9506>. Acesso em: 28 abr. 2015. DOI: 10.1080/17452007.2012.659506.

SUCCAR, B.; SHER, W.; WILLIAMS, A. An integrated approach to BIM competency assessment, acquisition and application. Automation in Construction, v. 35, p. 174-189, 2013. Disponivel em: <http://www.researchgate.net/ publication/256502957_An_integrated_ approach_to_BIM_competency_ assessment_acquisition_and application>. Acesso em: 30 abr. 2015. DOI: 10.1016/j.autcon.2013.05.016.

\section{Saulo Britto \\ SauloBritto.arq@gmail.com \\ Sérgio Scheer \\ scheer@ufpr.br \\ Calvin Kam \\ calvin.kam@stanford.edu \\ Martin Fischer \\ fischer@stanford.edu}

